\title{
Plasma Wave Wigglers for Free-Electron Lasers
}

\author{
CHAN JOSHI, MEMber, IEeE, T. KATSOUlEAS, J. M. DAWSON, Y. T. YAN, AND JACK M. SLATER
}

\begin{abstract}
We explore the possibility of using a relativistic plasma density wave as a wiggler for producing free-electron laser radiation. Such a wiggler is a purely electric wiggler with frequency $\omega_{p}$ (plasma frequency) and wavenumber $k_{p}$. If an electron beam is injected parallel to the plasma wave wavefront, it is wiggled transversely with an apparent wiggler wavelength $\lambda_{w}=2 \pi c / \omega_{p}$. Using plasma densities in the $10^{17}\left(\mathrm{~cm}^{-3}\right)$ range, $\lambda_{w}$ of order $100 \mu \mathrm{m}$ may be obtained, thereby permitting generation of short wavelength radiation with modest energy beams. The effective wiggler strength $a_{w}=e A / m c^{2} \sim 0.5$ can be extremely large. We discuss the excitation methods for such wigglers and examine the constraints imposed by the plasma medium on FEL gain in this scheme.
\end{abstract}

\section{INTRODUCTION}

$\mathrm{T}$ HERE is a great deal of interest in developing short wavelength wigglers for free-electron lasers and synchrotrons. Unfortunately, the high magnetic field strengths needed for such wigglers are not attainable with present technology for conventional static magnetic wigglers with wavelengths less than $1 \mathrm{~cm}$. Operation at wavelengths shorter than this, therefore, would require a large increase in the number of wiggler periods, and thus result in a very small extraction efficiency as well as require a very small electron beam energy spread and emittance. In this paper, we explore the suitability of purely electric wigglers with an effective wavelength in the $100 \mu \mathrm{m}$ range. Such wigglers, because of their extremely short wavelengths, allow the use of a rather modest energy electron beam for FEL action in the visible or VUV range.

In our proposal, the purely electric wiggler is a relativistic plasma density wave. The geometry of the plasma wiggler is illustrated in Fig. 1. In this simplified picture; the wiggler consists of a purely electric field oscillating perpendicular to the electron beam with a frequency $\omega_{p}$, but with no spatial dependence since $k$ of the radiation is transverse to $\boldsymbol{k}_{p}$. The amplification of radiation by such a field in vacuum has been modeled recently by Yan and Dawson [1]. Using a plasma wave as a purely electric wiggler is attractive for two reasons. First, the effective wiggler wavelength $\left(\sim 2 \pi c / \omega_{p}\right.$, typically of order 100 $\mu \mathrm{m}$ ) is shorter than that available with conventional magnet wigglers, and second, the effective wiggler strength

Manuscript received October 27, 1986; revised April 3, 1987. This work was supported by DOE Contract DE-AS03-ER 40120, NSF Grant ECS 83 10972, ONR Contract N00014-86-K-0584, and the Los Alamos National Laboratory.

C. Joshi and T. Katsouleas are with the Department of Electrical Engineering, University of California, Los Angeles, CA 90024.

J. M. Dawson and Y. T. Yan are with the Department of Physics, University of California, Los Angeles, CA 90024.

J. M. Slater is with Spectra Technology, Inc., Bellevue, WA 98004.

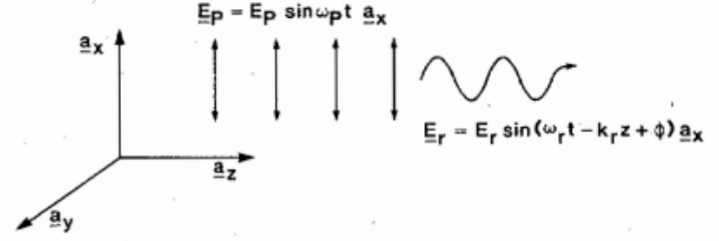

Fig. 1. Geometry of the plasma wiggler FEL.

can be extremely large (equivalent to $1 \mathrm{MG}$ wiggler fields at $100 \mu \mathrm{m}$ wavelength). However, when a plasma medium is used for exciting the purely electric wiggler, the FEL electron beam can be strongly influenced by it. In this paper, we discuss how plasma density waves suitable for FEL action can be excited and the limitations to FEL gain imposed by the plasma medium.

\section{FEL Mechanism in a Plasma Wiggler}

The equivalence among magnetic, electromagnetic, and purely electric (plasma) wigglers can most easily be seen from the point of view of the electrons. Fig. 2 illustrates the fact that although the three cases appear quite different in the laboratory frame, in the electron frame, all appear to be electromagnetic waves. Here, $\omega_{0}$ and $\omega_{p}$ represent the frequencies of the electromagnetic and electrostatic (plasma) waves, respectively, and $k_{0}$ and $k_{w}$ represent the wavenumbers of the electromagnetic and magnetic wigglers, respectively, in the laboratory frame. The purely electric wiggler has a zero $k$, whereas the purely magnetic wiggler has a zero $\omega$. In the electron frame, the momentum four vectors are

$$
\begin{aligned}
\left(\begin{array}{l}
k^{\prime} c \\
i \omega^{\prime}
\end{array}\right)=\left[\begin{array}{cc}
\gamma & i \beta \gamma \\
-i \beta \gamma & \gamma
\end{array}\right]\left(\begin{array}{c}
k_{0} \\
i \omega_{0}
\end{array}\right) \text { electromagnetic } \\
\left(\begin{array}{c}
k_{w} \\
0
\end{array}\right) \text { purely magnetic } \\
\left(\begin{array}{c}
0 \\
i \omega_{p}
\end{array}\right) \text { purely electric (plasma) } .
\end{aligned}
$$

For $\beta \simeq 1$, the transformed quantities satisfy $\omega^{\prime}=k^{\prime} c$ for all the cases, except that their wavelengths are different: $\lambda^{\prime}=\lambda_{w / \gamma}$ (magnetic), $\lambda_{0 / 2 \gamma}$ (electromagnetic), and $2 \pi c / \omega_{p} \gamma$ (purely electric or plasma). Each electromagnetic wave has a wiggler strength $a_{w}=e E^{\prime} / m \omega^{\prime} c=$ $e A / m c^{2}$ equal to its corresponding value, $e B \lambda_{w} / 2 \pi m c^{2}$ 


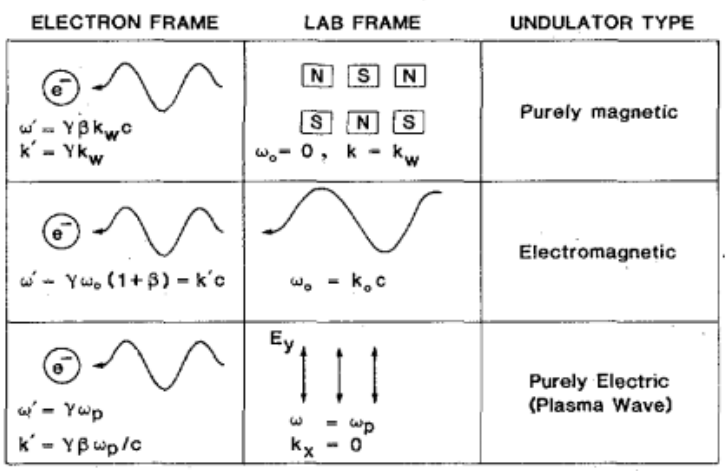

Fig. 2. Equivalence among magnetic, electromagnetic, and purely electric (plasma) wigglers.

(magnetic), $e E_{0} / m \omega_{0} c$ (electromagnetic), and $e E_{p} / m \omega_{p} c$ (purely electric or plasma) in the lab frame. In the plasma wiggler, as in the other two cases, the noise that seeds the lasing process comes from Compton scattering, with the radiated frequency in the exact forward direction $\omega_{r} \simeq$ $2 \gamma^{2} \omega_{p}$. Once there is spontaneous emission at $\omega_{r}$, there is a resonant ponderomotive force on the radiating electrons. This force is $\boldsymbol{F}=-e \boldsymbol{v}_{w} \times \boldsymbol{B}_{r} / c$ where $v_{w}$ is the wiggle velocity and $B_{r}$ is the magnetic field of the spontaneous or stimulated radiation. For a plasma wave (wiggler) of the form $\boldsymbol{E}=E_{p} \sin \left(\omega_{p} t\right) \boldsymbol{a}_{x}$ and radiation field

$$
\boldsymbol{B}_{r}=\frac{c k_{r}}{\omega_{r}} E_{r} \sin \left(k_{r} z-\omega_{r} t+\phi\right) \boldsymbol{a}_{y},
$$

the ponderomotive force is

$$
\begin{aligned}
\boldsymbol{F}= & \boldsymbol{a}_{z} \frac{1}{2}\left(\frac{e E_{p}}{\gamma m \omega_{p} c}\right)\left(\frac{e E_{r}}{m \omega_{r} c}\right) m c^{2} k_{r} \\
& \times\left\{\sin \left[k_{r} z-\left(\omega_{r}-\omega_{p}\right) t+\phi\right]\right. \\
& \left.+\sin \left[k_{r} z-\left(\omega_{r}+\omega_{p}\right) t+\phi\right]\right\} .
\end{aligned}
$$

In order to obtain a net energy exchange from the electron beam to the radiation, the electrons must have velocities in a range that is slightly greater than the phase velocity of the ponderomotive bucket. The second term in (2) has a phase velocity slightly greater than $c$, and thus time averages to zero. The phase velocity of the resonant (first) term in (2) is

$$
\begin{aligned}
v_{\phi} & =\frac{\omega_{r}-\omega_{p}}{k_{r}}=\frac{\omega_{r}}{k_{r}}\left(1-\frac{\omega_{p}}{\omega_{r}}\right) \\
& \simeq c\left(1-\frac{\omega_{p}}{\omega_{r}}+\frac{\omega_{p}^{2}}{2 \omega_{r}^{2}}\right) .
\end{aligned}
$$

The last term $\omega_{p}^{2} / 2 \omega_{r}^{2}$ in (3) arises because $\omega_{r} / k_{r}$ is not exactly $c$ in a plasma. However, for relativistic beams, $\omega_{p} / \omega_{r}<<1$, so that this term can be neglected. Thus, the resonance condition $\left(v=v_{\phi}\right)$ and $\gamma=(1-$ $\left.v^{2} / c^{2}\right)^{-1 / 2}$ becomes $\omega_{r} \leqslant 2 \gamma^{2} \omega_{p}$, in complete analogy to conventional FEL's.

The effective maximum undulator strength parameter $a_{w}$ of the plasma wiggler can be easily estimated from 1-D
Poisson's equation: $\nabla \cdot \boldsymbol{E}=-4 \pi e n_{1}$ where $n_{\mathrm{l}}$ is the perturbed electron density associated with the plasma wave. The maximum density rarefaction occurs when $n_{1}=n_{0}$, known as the cold plasma wavebreaking limit. In this limit, $\left|i k_{p} E_{p, \max }\right| \simeq 4 \pi e n_{0}$ or $\omega_{p} / k_{p} c=1$. Thus, the maximum value of $E_{p}$ attainable in the plasma corresponds to $a_{w}=1$, independent of the plasma density. The effective maximum magnet strength of a plasma wave is

$$
\begin{aligned}
B_{\text {eff }}(\text { gauss }) & =\frac{2 \pi m c^{2}}{e \lambda_{p}} a_{w} \leq \frac{2 \pi m c^{2}}{e \lambda_{p}} \\
& \simeq \frac{10^{4}}{\lambda_{p}(\mathrm{~cm})} \simeq 3 \times 10^{-3} \sqrt{n_{0}\left(\mathrm{~cm}^{-3}\right)} .
\end{aligned}
$$

It can be seen that effective magnetic field strengths of order $1 \mathrm{MG}$ are possible in a plasma of density $10^{17} \mathrm{~cm}^{-3}$.

The undulating mechanism of the plasma wiggler is shown schematically in Fig. 3. The electron beam is assumed to be thin; the diameter of the beam is small compared to $\lambda_{p} \simeq 2 \pi c / \omega_{p}$. In Fig. 3(a), the electric field $E_{p}$ of the plasma wave deflects the electrons toward the left of the figure where there is an excess of ions. Since both the electrons and the plasma wave are moving at the speed of light, by the time the electrons move up by a distance $\pi c / \omega_{p}$, the plasma wave moves to the right by the same amount. The electric field $E_{p}$ now drives the electron to the right. This is shown in Fig. 3(b). At a time $\pi / \omega_{p}$ later still, Fig. 3(c), the electrons move up another $\pi c / \omega_{p}$ while the plasma wave moves to the right by the same amount. The electric field $E_{p}$ once again drives the electrons to the left. Thus, the apparent wiggler wavelength is $\lambda_{w}=$ $2 \pi c / \omega_{p}$ which is the same as the plasma wave wavelength. The choice of plasma density determines the effective wiggler wavelength

$$
\lambda_{w}(\mathrm{~cm}) \simeq 3 \times 10^{6} / \sqrt{n_{0}}\left(\mathrm{~cm}^{-3}\right) .
$$

Thus, for plasma densities in the range $10^{13}-10^{18} \mathrm{~cm}^{-3}$, $\lambda_{w}$ ranges from $1 \mathrm{~cm}$ to $30 \mu \mathrm{m}$.

\section{Plasma Wiggler Excitation}

The relativistic plasma wave, used for a wiggler, can be excited by two schemes known as laser beat wave excitation [2] and wake field excitation [3]. Both are actively being investigated for accelerator applications in which the electrons are injected in the same direction as the phase velocity of the wave. If, instead, the electrons are injected perpendicular to the plasma wave (parallel to the wavefronts), they are wiggled transversely causing them to radiate.

In the beat wave excitation scheme, two laser beams $\left(\omega_{0}, k_{0}\right)$ and $\left(\omega_{1}, k_{1}\right)$ are copropagated into a homogeneous plasma such that their frequency difference is the plasma frequency. Under such resonant excitation, the radiation pressure of the lasers drives up the plasma wave, which eventually saturates due to relativistic effects [4]. The peak amplitude at saturation, assuming a square- 


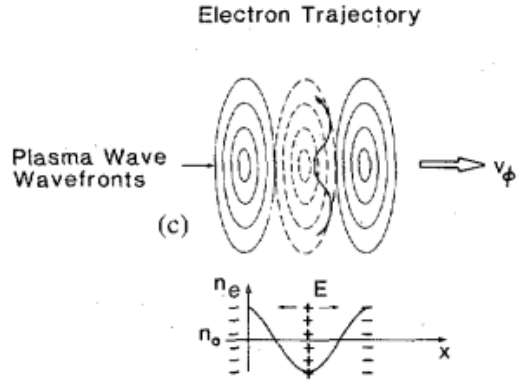

(b)

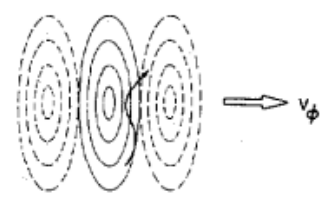

(a)
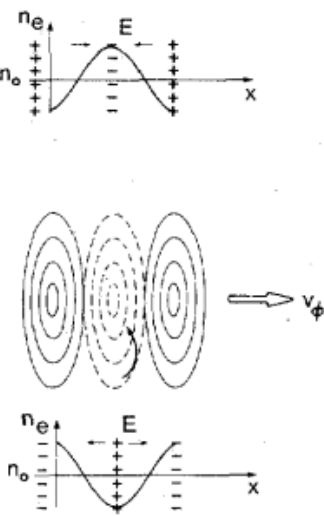

Fig. 3. Undulating mechanism of the plasma wiggler. Solid contours are increasing in density and dotted contours are decreasing in density compared to the background plasma density.

shaped laser pulse, is given by

$$
a_{w}^{\text {plasma }}=\left(\frac{16}{3} a_{w}^{0} a_{w}^{1}\right)^{1 / 3}<1
$$

where superscripts refer to the plasma wave and the two lasers and $a_{w}^{j}=e E^{j} / m \omega^{j} c$ as before. Note that, once again, the saturation amplitude is independent of the plasma density. The growth rate of the plasma wave, however, does depend on the plasma density. The excitation of plasma waves by this technique has been demonstrated in a series of experiments at UCLA [5]. Using a $\mathrm{CO}_{2}$ laser, with a modest intensity of $2 \times 10^{13} \mathrm{~W} / \mathrm{cm}^{2}$, operating on 10.6 and $9.6 \mu \mathrm{m}$ lines, a plasma wave with $a_{w} \sim 0.03-0.1$ was excited in a $10^{17} \mathrm{~cm}^{-3}$ density plasma. This corresponds to a $105 \mu \mathrm{m}$ period wiggler with an equivalent magnetic field of $\sim 32-95 \mathrm{kG}$. Using a more intense laser should allow even higher wiggler strengths to be generated.

Table I shows the possible combination of laser wavelengths that could be used to excite plasma wigglers of various wavelengths. The intensity required, in Table I, is for $a_{w}=0.5$ assuming that both lasers have squarewave shapes and equal intensities. The intensity required changes somewhat when one takes into consideration the risetime of the laser pulse [6].
We have carried out two-dimensional computer simulations to examine the transverse (to $k_{p}$ ) coherence of the plasma wave [2], [6]. In order to act as a wiggler, the potential contours of the plasma wave must be planar. The results of a typical $2 \mathrm{D}$ simulation, using a fully relativistic, electromagnetic particle code, are depicted in Fig. 2 . The simulation parameters were as follows: two laser beams $\omega_{0}=5 \omega_{p}$ and $\omega_{1}=4 \omega_{p}$ each with an $\operatorname{rms} a_{w}^{0,1}=$ 0.4 , a transverse intensity profile of $\cos ^{2} y$, and a beam width of $30 \mathrm{c} / \omega_{p}$ were injected into a homogeneous plasma $60 \mathrm{c} / \omega_{p}$ long and $60 \mathrm{c} / \omega_{p}$ wide with a temperature of $2.5 \mathrm{keV}$. The laser beams had a cubic rise from zero to maximum intensity in $300 / \omega_{p}$, after which the laser intensity remained constant. The ion-to-electron mass ratio was 1836 and the temperature ratio was 1 . Thus, for an IR laser with wavelengths 9.6 and $12 \mu \mathrm{m}$, the parameters were: a hydrogen plasma with $n_{e} \sim 4.9 \times$ $10^{17} \mathrm{~cm}^{-3}, \lambda_{w} \sim 47 \mu \mathrm{m}$, laser risetime $\sim 7.6 \mathrm{ps}$, laser intensity $\sim 2 \times 10^{15} \mathrm{~W} / \mathrm{cm}^{2}$, beam width $\sim 25$ wavelengths, and system length 50 wavelengths. The simulation parameters were chosen to study both the growth and saturation of the plasma wave and its transverse coherence and stability.

In Fig. 4(a), the laser beat wave contours at $150 / \omega_{p}$ are shown as the beams propagate from left to right. Fig. 4(b) shows the contour plot of the potential of the plasma density wave at the same time. According to the fluid theory, the plasma wave should reach peak amplitude at this time. The plasma wavefronts in the contour plot are seen to be planar. A section through the center of the transverse axis of the longitudinal electric plot is shown in Fig. 4(c). An extremely coherent plasma wave builds up rapidly in time/ space and saturates at $a_{w} \simeq 0.5$, in excellent agreement with the value expected from the fluid theory [4]. We find that the plasma wave typically remains coherent for a few ion plasma periods, which translates to between a few to a few tens of picoseconds for parameters in Table I before disrupting due to ion dynamics.

In this scheme, we need a wide plasma wave which has $E_{z}$ nearly constant over 100 or so wiggle periods. The actual length of the wave (in the direction of the driving lasers) need only be two or three wavelengths with the central potential trough used for wiggling the electron beam. The laser beam pump depletion length is given by [7]

$$
L_{d} \geq \frac{\omega_{0}^{2}}{\omega_{p}^{2}} \frac{c}{\omega_{p}} \frac{4}{a_{w}^{\text {plasma }}} .
$$

This assumes that the maximum effective length of the laser pulse is determined by relativistic detuning [4]. For parameters in Table I, $\omega_{0} / \omega_{p} \geq 10$ and $a_{w}^{\text {plamsa }} \sim 0.5, L_{d}$ can be hundreds of plasma wavelengths long. Thus, by using small $F$ number cylindrical focus optics which produce a line focus that is greater than $100 \lambda_{w}$ wide but only has a Rayleigh length of about $3 \lambda_{w}$, it may be possible to reuse the laser light.

We now briefly discuss an alternate method of excitation of the plasma wiggler which uses a high-current but 
TABLE I

Parameters for Exciting Pl.asma Wigglers of Different Wavelengths

\begin{tabular}{lcccc}
\hline \hline Laser Type & $\begin{array}{c}\text { Laser } \\
\text { Wavelengths } \\
(\mu \mathrm{m})\end{array}$ & $\begin{array}{c}\text { Intensity } \\
\left(\mathrm{W} / \mathrm{cm}^{2}\right)\end{array}$ & $\begin{array}{c}\text { Wiggler } \\
\text { Wavelength } \\
(\mu \mathrm{m})\end{array}$ & $\begin{array}{c}\text { Approximate } \\
\text { Plasma Density } \\
\left(\mathrm{cm}^{-3}\right)\end{array}$ \\
\hline $\mathrm{CO}_{2}$ & 9.6 and 10.6 & $2 \times 10^{14}$ & 100 & $10^{17}$ \\
$\mathrm{CO}_{2}$ & 9.6 and 10.3 & $2 \times 10^{14}$ & 140 & $5.8 \times 10^{16}$ \\
$\mathrm{CO}_{2}$ & 10.6 and 10.3 & $2 \times 10^{14}$ & 364 & $10^{16}$ \\
$\mathrm{Nd}$ :glass & 1.05 and 1.06 & $2 \times 10^{16}$ & 110 & $10^{17}$ \\
\hline
\end{tabular}

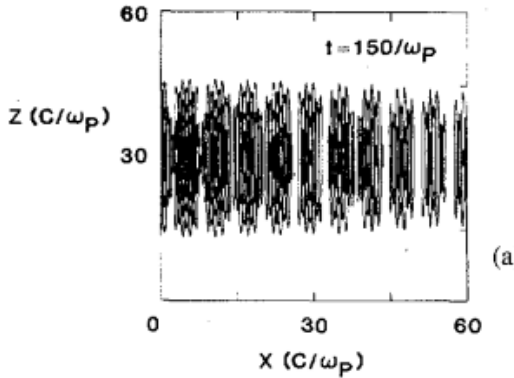

(a)

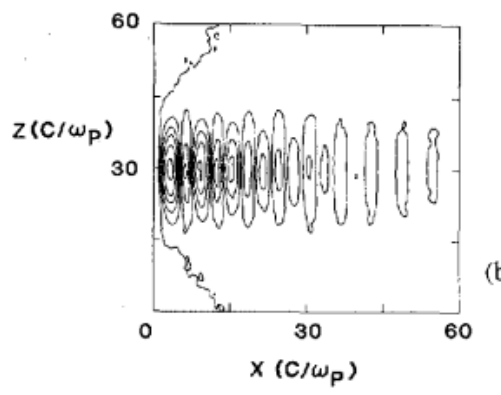

(b)

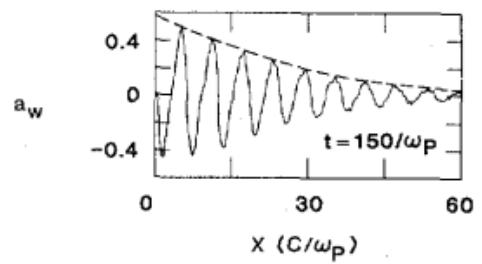

Fig. 4. Laser beat excitation of plasma wiggler. (a) Laser beat contours as lasers propagate from left to right, (b) wavefronts of the relativistic plasma wave, and (c) longitudinal electric field or, equivalently, $a_{w}$ on axis. The dotted line is the predicted value of the $a_{w}$ on axis from fluid theory [4].

low-voltage electron beam as the free energy source. This is known as the wake field excitation scheme. To distinguish this electron beam from the radiating beam, we shall call this the driver bunch. As shown by Chen et al. [3], when such a driver bunch is shot through a high-density plasma, the space-charge force of the bunch displaces the plasma electrons and leaves behind a wake of plasma oscillations. The phase velocity of the wake, like that of a wake behind a boat, is tied to the velocity of the driving bunch, which is almost $c$. The undulating mechanism in this scheme is exactly the same as in the laser beat wave scheme. In order to obtain a large $a_{w}$, one has to use a driving bunch that is much smaller than $\lambda_{p}$ ( say $\pi c / \omega_{p}$ ). For $a_{w}$ of up to 0.5 , provided that the above condition is met, then $a_{w} \sim n_{b} / n_{0}$ where $n_{b}$ is the driving beam den- sity. The required beam current is $I_{b}$ (amps) $=10^{3} \mathrm{~N}$ $\left(n_{b} / n_{0}\right)$ if the driving bunch is assumed to be like a football, $N c / \omega_{p}$ wide in one dimension and $c / \omega_{p}$ wide in the other.

As an example, consider a plasma wake field wiggler excited by a $5 \mathrm{ps}$ long bunch from a linac. The wiggler wavelength and strength turn out to be $3 \mathrm{~mm}$ and $15 \mathrm{kG}$, respectively, if the plasma density is $10^{14} \mathrm{~cm}^{-3}$. Unfortunately, to excite a wave that is 100 wiggler periods wide, we need $50 \mathrm{kA}$ current. Thus, at the present time, this method of excitation for the plasma wave wiggler does not seem as promising as the laser beat wave method.

Two-dimensional simulations of the wake field excitation of a plasma wave have been carried out [8] which show the same qualitative behavior regarding the transverse coherence and stability as that of a plasma wave excited by a laser beat wave.

\section{FEL Parameters for Gain}

As mentioned earlier, the plasma wiggler in principle is exactly the same as a static magnetic wiggler of comparable wavelength and strength. To the zeroth order, the requirements for obtaining a significant gain are exactly the same for the plasma wiggler as that for a static magnetic wiggler as far as the radiating electron beam is concerned. Here we work out some typical parameters assuming that we have a wiggler with 100 wiggle periods with $\lambda_{w}$ of $100 \mu \mathrm{m}$ and an $a_{w}$ of 0.5 . We work out beam parameters for obtaining a single pass gain of 20 .

We start with the maximum small-signal gain formula for an FEL driven by a perfectly monoenergetic beam [9]:

$$
G \sim 2 \times 10^{-3} \frac{a_{w}^{2}}{\left(1+a_{w}^{2}\right)} N^{2} I / \gamma
$$

where the optimum beam cross section $\sigma=N \lambda_{R} \lambda_{w} / \sqrt{3}$ was assumed. The FEL beam parameters consistent with the energy spread requirement $\Delta \gamma / \gamma=1 /(2 N)$ and divergence angle $\Delta \theta<1 /(\gamma \sqrt{N})$ turn out to be as shown in Table II.

The gain formula provides valuable insight, but is not quantitatively reliable. By not taking into account the change in the amplitude of the radiation field, it underestimates the actual gain; by neglecting the energy spread, it overestimates the gain.

The angular contribution to the energy spread is $(\Delta \gamma / \gamma)_{\epsilon} \sim \gamma^{2} \Delta \theta^{2}$. The emittance consistent with this is $\pi r \Delta \theta$ which, for the parameters listed in Table II, is be- 
TABLE II

PARAMETERS For ObTAINING Gain

\begin{tabular}{ccccccc}
\hline \hline $\begin{array}{c}\text { Beam Energy } \\
(\gamma)\end{array}$ & $\begin{array}{c}\text { Radiated } \\
\text { Wavelength } \\
\lambda_{r}(\mu \mathrm{m})\end{array}$ & $\begin{array}{c}\text { Beam } \\
\text { Current } \\
(\mathrm{A})\end{array}$ & $\begin{array}{c}\text { Beam } \\
\text { Diameter } \\
(\mu \mathrm{m})\end{array}$ & $\begin{array}{c}\text { Beam } \\
\text { Density } \\
\left(\mathrm{cm}^{-3}\right)\end{array}$ & $\begin{array}{c}\text { Divergence } \\
\text { Angle } \\
\theta(\mathrm{rad})\end{array}$ & $\begin{array}{c}\text { Beam Brightness } \\
\left(\mathrm{A} \cdot \mathrm{cm}^{-2} \cdot \mathrm{rad}^{-2}\right)\end{array}$ \\
\hline 15 & 0.22 & 75 & 36 & $1.5 \times 10^{15}$ & $6.7 \times 10^{-3}$ & $2.3 \times 10^{8}$ \\
20 & 0.125 & 100 & 27 & $3.6 \times 10^{15}$ & $5 \times 10^{-3}$ & $5.6 \times 10^{8}$ \\
50 & 0.02 & 250 & 11 & $5.4 \times 10^{16}$ & $2 \times 10^{-3}$ & $8.3 \times 10^{9}$ \\
\hline
\end{tabular}

tween $0.76-0.07 \mathrm{~mm} \cdot \mathrm{mrad}$. There is another constraint on the electron beam emittance. An electron entering the wiggler at an angle $\theta$ to the beam axis has a displacement $N \lambda_{w} \Delta \theta$ after it has traversed the length of the wiggler. Unless the displacement is much smaller than the plasma wave wavelength, a significant fraction of the electrons will absorb power from the electromagnetic radiation. Thus, letting $N \lambda_{w} \Delta \theta<\lambda_{w} / 10$, we have for $N=100, \theta$ $=1 \mathrm{mrad}$, and $\epsilon=0.1-0.035 \mathrm{~mm} \cdot \mathrm{mrad}$ for the parameters listed in Table II. Such high-quality beams will be required to obtain the FEL action in the short wavelength regime no matter what kind of wiggler is used. The plasma medium imposes some additional restrictions on the electron beam which will be discussed shortly.

Detailed calculations and simulations of the plasma wiggler FEL which take into account the variation in $a_{w}$ across the beam cross section, edge effects, beam energy spread, emittance, and plasma wave harmonics are being carried out presently. The rest of this paper is devoted to discussing the influence of the plasma medium on the radiating electron beam.

\section{Plasma Effects on the Radiating Electron BEAM}

Thus far in this and other plasma-based FEL schemes [10], the effect of the plasma medium on the FEL process has been neglected. In this section, we consider the consequences of beam-plasma interactions for FEL gain and the effect of the plasma in altering the resonance condition. The main concern with beam-plasma interactions is that they may degrade the electron beam quality (i.e., energy spread and emittance) beyond the acceptable limits for FEL gain. Such degradation can arise either from plasma instabilities or plasma wave fields created by the beam. The plasma can also modify the resonance condition because $V_{p h}>c$ for light waves in plasma.

For sufficiently small beams, plasma instabilities may be avoidable. Consider the well-known two-stream instability. This results in the growth of plasma space-charge oscillations on the beam with frequency $\omega \simeq \omega_{p}$ and parallel wavenumber $k_{z} \geq \omega_{p} / v_{b}$ where $v_{b} a_{z}$ is the beam velocity. The growth rate of the instability for relativistic beams is $\nu \sim\left(n_{b} / n_{0}\right)^{1 / 3} \omega_{p} / \gamma$ and the group velocity is $v_{g} a_{z} \simeq(2 / 3) c$. If the $e$-folding length of the instability $\left(v_{g} a_{z}\right) / \nu$ is greater than the length $L$ of the beam, then the two-stream instability cannot grow. Thus, we expect to avoid this instability using beams of length

$$
L \lesssim \gamma\left(n_{0} / n_{b}\right)^{1 / 3} c / \omega_{p} .
$$

For instance, a $10 \mathrm{MeV}$ beam in a $10^{16} \mathrm{~cm}^{-3}$ plasma should be of order $1 \mathrm{~mm}(\sim 3 \mathrm{ps})$ long or less.

Another instability of concern is the Weibel instability. This purely transverse $\left(a_{x}\right.$ or $\left.a_{y}\right)$ instability is responsible for filamentation of wide beams in plasmas and is not suppressed by short bunches. Fortunately, it is largely suppressed by beams that are narrower then the plasma skin depth $c / \omega_{p}$. However, we have already assumed that we have beams that are narrower than this in preceding discussion.

For sufficiently short and narrow beams, it is the beams' own wake fields rather than instabilities which are important. The radial and longitudinal wake forces on a relativistic, uniform beam of length $L$, charge $-e N_{e}$, and radius $a$ ( less than $\left.c / \omega_{p}\right)$ are approximately [11]

$$
\begin{aligned}
F_{r}= & -e\left(E_{r}-B_{\theta}\right)=-\frac{4 \pi e^{2} N_{e} k_{p}}{L} \frac{r}{2 \pi k_{p} a^{2}} \\
& \cdot\left[1-\cos k_{p}(z-c t)\right] \theta(t-z / c) \\
F_{z}= & -e E_{z}=\frac{4 \pi e^{2} N_{e} k_{p}}{L} \\
& \cdot \sin \left[k_{p}(z-c t)\right] \theta(t-z / c)
\end{aligned}
$$

where $-z$ is the distance behind the leading edge of the beam, $r$ is radial position, and $\theta$ is the step function. In terms of convenient units, the common coefficient $\left(4 \pi e^{2} N_{e} k_{p} / L\right)$ can be expressed as

$$
\left|F_{z}(\mathrm{MeV} / \mathrm{cm})\right|=7 \times 10^{-10} I[\mathrm{amps}] \sqrt{n_{0}\left(\mathrm{~cm}^{-3}\right)}
$$

where the beam radius in $\mathrm{cm}$ is assumed less than $\mathrm{c} / \omega_{p}(\mathrm{~cm})$.

The radial force $F_{r}$ gives rise to emittance growth, while the longitudinal force $F_{z}$ directly gives rise to energy spread. The radial wake exerts a pinching force on the beam. This effect is used to advantage in the plasma lens scheme for focusing electron beams [12]. Here, the pinching leads to undesirable angular variation (i.e., emittance growth) of the beam particles. A physical explanation of the pinching force in the plasma is as follows. In vacuum, the radial space-charge repulsion of a relativistic beam is cancelled [ to order $\left(1-v_{b} / c\right)$ ] by the $v_{z} \times B_{\theta}$ force created by the beam's own azimuthal 
magnetic field. In plasma, the space charge of the beam expels plasma electrons, leaving plasma ions to neutralize the space-charge repulsion of the beam. However, the plasma does not completely neutralize the beam current ( since the return current flows in a cylinder of radius $c / \omega_{p}$, which is partly outside of the assumed beam radius). The net result is the pinching of the beam by the $v_{z} \times B_{\theta}$ force.

Quantitatively, the angular spread of the beam can be estimated from the betatron motion of individual particles. These transverse oscillations are described approximately by the harmonic equation

$$
\frac{\gamma m d^{2} r}{d t^{2}}=F_{r} \simeq\left(\frac{F_{r}}{r}\right) r \simeq \gamma m c^{2} \frac{d^{2} r}{d z^{2}} .
$$

Thus, the betatron wavelength is approximately $\beta \simeq$ $2 \pi\left(\gamma m c^{2} r /-F_{r}\right)^{1 / 2}$. The betatron oscillations give rise to an angular spread of the beam of order $(\Delta \theta)_{\beta} \simeq 2 \pi r / \beta$. For the parameters of Table II, this gives $(\Delta \theta)_{\beta}$ in the range $2.4 \times 10^{-3} \mathrm{rad}(\gamma=15)-7.46 \times 10^{-4} \mathrm{rad}(\gamma=$ 50 ). Since focusing force rises quadratically from zero at the end of the bunch, short bunches with $k_{p} L<<1$ will have smaller angular spreads by a factor $k_{p} L / 2$. The minimum beam length for FEL gain is $L=N \lambda_{r} \simeq N \pi / k_{p} \gamma^{2}$. For the present parameters, this gives $k_{p} L / 2=N / 2 \gamma^{2}$ and $(\Delta \theta)_{\beta}=1.67 \times 10^{-3} \mathrm{rad}(\gamma=15)-4.7 \times 10^{-5} \mathrm{rad}$ $(\gamma=50)$ which is small enough to allow gain.

The longitudinal wake directly gives rise to energy spread. Simple uniform beams in plasmas will always suffer unacceptable energy spread for FEL action, as can be seen from the following argument. The energy spread over the length of the FEL is of order $\Delta \gamma m c^{2} \simeq\left|F_{z}\right| \cdot N \lambda_{w}$. Substituting from (10) and (5), this becomes $\Delta \gamma \simeq 5 \times$ $10^{-3} N I$ [amps]. However, $\Delta \gamma / \gamma \simeq 1 / 2 N$ for gain, so $5 \times 10^{-3} \mathrm{NI} / \gamma$ has to be less than $1 / 2 \mathrm{~N}$ or

$$
N^{2} I / \gamma \leq 100
$$

Since the gain $G$ is less $\sim 2 \times 10^{-3} N^{2} I / \gamma(8)$, we find that $G \leq 0.02$.

In order to obtain a reasonable single-pass gain of, say (20), one must reduce the longitudinal wake force by a factor 100. One solution is ultrashort beams. From (10), the peak retarding force scales as $k_{p} L$ for $k_{p} L<<1$, so that beam length would need to be less than $0.1 k_{p}^{-1}=$ $0.01 \mathrm{c} / \omega_{p} \simeq 5 \times 10^{3} / n_{0}\left(\mathrm{~cm}^{-3}\right) \mathrm{cm}$. Another solution is to employ a precursor beam [8]. This had been suggested as a means of providing uniform focusing in the plasma lens [2] scheme. It can be shown that any precursor arrangement that provides uniform focusing (i.e., uniform radial wake force within the beam) also creates a longitudinal wake which exactly cancels the trailing beam's longitudinal wake. This eliminates the energy spread arising from longitudinal wake fields.

For example, the longitudinal energy spread could be avoided if one could inject a short precursor bunch ahead of the FEL beam by a distance $\lambda_{p} / 4+m \lambda_{p}(m=0,1$, $2, \cdots)$, with the amount of charge $-e N_{e} / k_{p} L$ where the
FEL beam length $L$ can now be arbitrarily long (subject to the two-stream instability).

In addition to affecting the electron beam, the plasma also affects the radiation. The phase velocity of the electromagnetic waves in the plasma is $c\left(1-\omega_{p}^{2} / \omega^{2}\right)^{-1 / 2}$, which is greater than $c$. This forces a restriction on the maximum allowable wiggler wavelength for which the ponderomotive buckets can be resonant with the electron beam. For example, consider the recently proposed scheme to use the self-generated magnetic fields of a series of laser-produced plasmas $\left(n_{0} \simeq 10^{20} \mathrm{~cm}^{-3}\right)$ as a static undulator [10]. In this case, the resonance condition is

$$
\begin{aligned}
v_{\phi} & \simeq \frac{\omega_{r}}{k_{r}+k_{w}} \simeq \frac{\omega_{r}}{k_{r}}\left(1-\frac{k_{w}}{k_{r}}\right) \\
& \simeq c\left(1-\frac{k_{w}}{k_{r}}+\frac{\omega_{p}^{2}}{2 \omega_{r}^{2}}\right)
\end{aligned}
$$

where $k_{w}$ is $\pi$ divided by the laser spot spacing ( $\sim 100$ $\mu \mathrm{m}$ in the example proposed). In order for this to be less than $c$, we require that $k_{w} / k_{r}>\omega_{p}^{2} / 2 \omega_{r}^{2}$ or

$$
k_{w}>\frac{1}{2 \gamma} \frac{\omega_{p}}{c} .
$$

For the static plasma wiggler parameters proposed by Loeb and Eliezer [10], $k_{w} \simeq \pi / 100 \mu \mathrm{m} \simeq 300 \mathrm{~cm}^{-1}$ and $\omega_{p} / 2 c \simeq 10^{4} \mathrm{~cm}^{-1}$, so $\gamma>30$ would be required. For the plasma wave wigglers described in this paper, the effective $k_{w}$ is $\omega_{p} / c$ so that this condition (14) is automatically satisfied for all relativistic beams.

\section{PRospects}

In this paper, we have introduced the concept of a plasma wave wiggler, which simultaneously offers the prospect of a small effective wiggler wavelength and a high wiggler strength. We have described how such wigglers may be produced and some limitations on the FEL process imposed by the plasma medium. There is a considerable ongoing effort in generating coherent relativistic plasma wave wavefronts for high-energy accelerator applications. A natural extension of this work will lead to characterization of these waves for wiggler application. Extremely high-quality electron beams are required for FEL action in the visible or VUV region using any kind of wiggler, including a plasma wave wiggler. Finally, although lasing may not be possible at even shorter wavelengths than this, the strong $a_{w}$ and short $\lambda_{w}$ of a plasma wiggler may enable significant spontaneous emission (say of $\gamma$ rays) to be obtained by using a modest energy electron beam (such as that from a compact $\mathrm{GeV}$ storage ring).

\section{ACKNOWLEDGEMENT}

We wish to thank W. Mori for the 2D simulations.

\section{REFERENCES}

[1] Y. T. Yan and J. M. Dawson, "ac free electron laser," Phys. Rev. Lett., vol. 57, pp. 1599-1602, 1986. 
[2] C. Joshi, W. B. Mori, T. Katsouleas, J. M. Dawson, J. M. Kindel and D. W. Forslund, "Ultrahigh gradient particle acceleration by intense laser-driven plasma density waves," Nature, vol. 311, pp. 525$529,1984$.

[3] P. Chen, J. M. Dawson, R. Huff, and T. Katsouleas, "Acceleration of electrons by the interaction of bunched electron beam with a plasma,"' Phys. Rev. Lett., vol. 54, pp. 693-696, 1985.

[4] M. N. Rosenbluth and C. S. Liu, "Excitation of plasma waves by two laser beams," Phys. Rev. Lett., vol. 29, pp. 701-704, 1972.

[5] C. E. Clayton, C. Joshi, C. Darrow, and D. Umstadter, "Relativistic plasma wave excitation by collinear optical mixing," Phys. Rev. Lett., vol. 54 , pp. 2343-2346, 1985.

[6] D. W. Forslund, J. M. Kindel, W. B. Mori, C. Joshi, and J. M. Dawson, "Two-dimensional simulations of single frequency and beat wave laser plasma heating," Phys. Rev. Lett., vol. 54, pp. 558-561, 1985.

[7] T. Katsouleas, C. Joshi, J. M. Dawson, F. F. Chen, C. E. Clayton, W. B. Mori, C. Darrow, and D. Umstadter, "Plasma accelerators," in Proc. Workshop on Laser Acceleration of Particles, Malibu, CA, AIP Conf. Proc. 130, C. Joshi and T. Katsouleas, Eds., 1985, pp. 63-98.

[8] T. Katsouleas, "Physical mechanisms in the plasma wake field accelerator,'” Phys. Rev. A, vol. 33, pp. 2056-2064, 1986.

[9] J. M. J. Madey, "Stimulated emission of Bremsstrahlung in a periodic magnetic field,"' J. Appl. Phys., vol. 42, p. 1906, 1971; W. B. Colson and A. M. Sessler, "Free electron lasers," Ann. Rev. Nucl. Part. Sci., vol. 35, pp. 25-54, 1985; P. Sprangle, R. A. Smith, and V. L. Granastein, "Free electron lasers and stimulated scattering from relativistic electron beams," in Infrared and Millimeter Waves, vol. 1, K. J. Button, Ed. New York: Academic, 1979, pp. 279-297; Y. Carmel, V. Granastein, and A. Gover, "Demonstration of a two-stage backward-wave-oscillator free-electron laser," Phys. Rev. Lett., vol. 51 , p. 566, 1983; L. R. Elias, "High power, cw, efficient, tunable (uv through ir) free-electron laser using low-energy electron beams," Phys. Rev. Lett., vol. 42, p. 977, 1979; I. Kimel, L. R. Elias, and G. Ramian, "Two stage free-electron laser driven by an electrostatic accelerator," Bull. Amer. Phys. Soc., vol. 32, p. 212, 1987; P. Dobiasch, P. Meystre, and M. Scully, "Optical wiggler free electron $\mathrm{X}$-ray laser in the $5 \AA$ region," IEEE J. Quantum Electron., vol. QE-19, pp. 1812-1820, 1983.

[10] A. Loeb and S. Eliezer, "Free electron laser and laser electron acceleration based on megagauss magnetic fields in laser-produced plasmas," Phys. Rev. Lett., vol. 56, p. 2252, 1986.

[11] T. Katsouleas, C. Joshi, and W. B. Mori, "Comment on free electron laser and laser electron acceleration based on megagauss magnetic fields in laser-produced plasmas,"'Phys. Rev. Lett., vol. 57, p. 1960, 1986; T. Katsouleas, "Beam loading in plasma accelerators," UCLA PPG-952, Particle Accelerators, 1986.

[12] P. Chen, J. J. Su, T. Katsouleas, S. Wilks, and J. M. Dawson, "Plasma focusing for high energy beams," IEEE Trans. Plasma Sci., Special Issue on Plasma Based High Energy Accelerators, 1987, to be published.

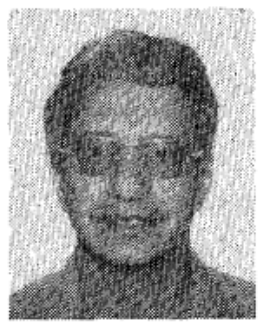

Chan Joshi (M'83) was born in India in 1953. He received the B.S. degree in nuclear engineering from London University, London, England, in 1974, and the Ph.D. degree in applied physics and from Hull University, England, in 1978.

He held a postdoctoral position at the National Research Council in Ottawa, Canada for two years. Since 1980 he has been with the Department of Electrical at UCLA; first as a member of the Research Staff and currently as an Associate Professor-in-residence. He teaches both undergraduate and graduate courses in electromagnetics, and quantum electronics. He has published over 60 technical papers. His research interests are in the areas of collective particle accelerators, laser-plasma interactions / nonlinear optics, development of X-ray CCD's, and ultrahigh power lasers.

Dr. Joshi is a member of the American Association for the Advancement of Science, the New York Academy of Sciences, and the American Physical Society.

T. Katsouleas, photograph and biography not available at the time of publication.

J. M. Dawson, photograph and biography not available at the time of publication.

Y. T. Yan, photograph and biography not available at the time of publication.

Jack M. Slater, for a photograph and biography, see this issue, p. 1513. 